www.nature.com/clinicalpractice/uro

prostatitis-chronic pelvic pain syndrome who had not received previous treatment with alpha-blockers. Participants were randomly assigned to receive either $10 \mathrm{mg}$ alfuzosin $(n=138)$ or placebo $(n=134)$ daily for 12 weeks. All men were assessed for symptoms and monitored for adverse events at baseline, week 6 and week 12. Symptoms were scored according to the National Institutes of Health Chronic Prostatitis Symptom Index (NIH-CPSI; score range $0-43$, higher scores indicate more severe symptoms).

Of the 233 patients who completed the study, $49.3 \%$ in both groups $(0.1 \%$ difference between groups, $95 \% \mathrm{Cl}-11.2$ to 11.0 ) had $a \geq 4$-point decrease in symptom score (the minimum clinically significant difference). Additionally, no significant differences were observed in adverse event rates.

Nickel et al. do not rule out the possibility that a longer treatment time with alfuzosin or use of another alpha-blocker might confer a beneficial effect, but suggest that alternative treatment options be considered for initial therapy of chronic prostatitis-chronic pelvic pain syndrome.

Original article Nickel JC et al. (2008) Alfuzosin and symptoms of chronic prostatitis-chronic pelvic pain syndrome. N Engl J Med 359: 2663-2673

\section{Single-port transvesical enucleation of the prostate: initial reports are positive}

A preliminary study has revealed that a minimally invasive single-keyhole surgery technique is technically feasible for the removal of large prostates. Desai and colleagues report on the initial outcomes of single-port transvesical enucleation of the prostate as an alternative method to invasive surgical enucleation of prostates greater than $80 \mathrm{~g}$ in size.

The procedure was performed in three patients aged 82,70 , and 78 years with largevolume benign prostatic hyperplasia (prostate sizes 187,93 , and $92 \mathrm{~g}$, respectively). A novel single-port device was used to access the bladder via a small incision $(2.5 \mathrm{~cm})$. The prostate adenoma was enucleated and divided intravesically into two or three pieces to allow removal through the incision.

Removal of the prostatic adenoma was successful in all three patients. A bowel injury occurred in the first patient during insertion of the single-port device near the umbilicus. This injury was repaired after enucleation. The incision was made between the umbilicus and symphysis in the second and third patients. After catheter removal (within 8 days of the procedure), all patients were able to urinate without difficulty and none suffered from incontinence.

Desai et al. highlight a number of theoretical advantages of single-port transvesical enucleation over surgical removal of the prostate: the whole adenoma can be enucleated and removal of the adenoma is easy; the risk of urethral strictures is minimized as urethral instrumentation is minimal; the absence of irrigation fluid potentially reduces the risk of infection; and bleeding can be monitored by means of a small endoscope.

Original article Desai MM et al. (2009) Single-port transvesical simple prostatectomy: initial clinical report. Urology 72: 960-965 